\title{
Zweitteuerstes Gesundheitswesen der Welt
}

G. Kocher

Die USA haben die weitaus höchsten Gesundheitsausgaben der Welt, gefolgt von der Schweiz. Dies gilt sowohl für den Anteil der Gesundheitsausgaben am Bruttoinlandsprodukt als auch für die Pro-Kopf-Ausgaben in kaufkraftbereinigten US-Dollars.

Korrespondenz:

Dr. rer. pol. Gerhard Kocher

Haldenweg 10a

CH-3074 Muri b. Bern

Tel. 0319526707

Fax 0319526800

E-Mail: kocher@spectraweb.ch
Die wichtigste Datenbasis für internationale Kostenvergleiche der Gesundheitssysteme ist die alljährliche CD-ROM «OECD Health Data». Die 13. Ausgabe 2004 dieser umfassenden Datenbank ist im Juni erschienen [1]. Sie enthält 550000 Zahlen in über 1200 Statistiken und Indikatoren mit vielen Zeitreihen von 1970 bis 2001 und teilweise 2002. Herausgegeben wird die CD-ROM von der Organisation für wirtschaftliche Zusammenarbeit und Entwicklung OECD in Paris. Diese Organisation produziert die zuverlässigsten Vergleichsstatistiken über die Gesundheitsausgaben ihrer 30 Mitgliedsländer.

\section{Gesundheitsausgaben gemessen am BIP}

Im Jahr 2002 beliefen sich die Schweizer Gesundheitsausgaben gemäss OECD auf Fr. 47,959 Milliarden (Fr. 5,5 Millionen pro Tag) oder Fr. 6579 pro Einwohner und Jahr. Dies entspricht 11,2\% (Vorjahr 10,9\%) des Bruttoinlandsprodukts (BIP). Nur die USA gaben mit 14,6\% noch (erheblich) mehr für ihr Gesundheitssystem aus (Tab. 1). An dritter Stelle kommt Deutschland (10,9\%), gefolgt von Island $(9,9 \%)$, Frankreich (9,7\%) und Kanada (9,6\%). Im 2. Rang war die Schweiz auch 2001; von 1995 bis 2000 hatte sie immer die dritthöchsten Ausgaben nach den USA und Deutschland.

Im Durchschnitt der von der OECD für 2002 erfassten 26 Industrieländer betragen die Ausgaben wie im Vorjahr 8,6\% des BIP. Die Gesundheitsausgaben der Schweiz sind 33\% höher. Die USA mit dem traditionell weitaus teuersten Gesundheitswesen der Welt geben sogar 73\% mehr aus als der OECD-Durchschnitt. Unter diesem Mittel sind unter anderen Italien $(8,5 \%)$, Österreich (7,7\%), Grossbritannien (7,7\%), Spanien $(7,6 \%)$ und Dänemark (7,3\%). Die Schweiz ist seit 1999 jeweils im zweiten Rang, vorher war es meist Deutschland.

Von 2001 auf 2002 konnte kein Land den Anteil am BIP senken. In Portugal blieb er gleich
(9,3\%). In allen anderen Ländern stieg er, im OECD-Durchschnitt um 4,9\%, nämlich von 8,2 auf $8,6 \%$ des BIP. Bei diesen Zahlen (Ausgaben im Verhältnis zum Bruttoinlandsprodukt) ist zu beachten, dass der Prozentsatz selbstverständlich nicht nur von den Gesundheitsausgaben, sondern auch von der wirtschaftlichen Entwicklung des Landes (gemessen am BIP) abhängt.

Als «dramatisch» und «alarmierend» bezeichnet die OECD in einer Medienmitteilung vom Juni 2004 den Anstieg der Gesundheitsausgaben in den OECD-Ländern. Von 1992 bis 1997 stiegen sie jährlich um 2,5\% - nur unwesentlich stärker als das Bruttoinlandsprodukt mit 2,4\%. Ganz anders von 1997 bis 2002: Gesundheitsausgaben $+4,3 \%$, BIP nur $+2,5 \%$ pro Jahr. Die Gesundheitsausgaben wuchsen in der Schweiz in diesen fünf Jahren im Vergleich zu den anderen OECD-Ländern zwar unterdurchschnittlich, um 3,2\%. In der Rangliste des BIPWachstums kam unser wachstumsschwaches Land aber nur auf Rang 27 von 30 Ländern: mit $1,2 \%$ jährlich im Vergleich zum OECD-Durchschnitt von $2,5 \%$.

\section{Ausgabenwachstum seit 1992}

Aufschlussreich ist auch ein Vergleich 1992 bis 2002 der Ausgaben für das Gesundheitswesen. Drei Länder von 25 konnten in dieser Periode den Anteil am Bruttoinlandsprodukt senken (Finnland um 19,8\%, Kanada um 4\% und Polen um 1,6\%), in Luxemburg blieb er unverändert, und in 21 Ländern stieg er um durchschnittlich 11,2\%: von 7,7\% im Jahr 1992 auf 8,6\% im Jahr 2002. Das grösste Wachstum verzeichneten die Tschechische Republik (37\%) und Portugal $(32,9 \%)$, gefolgt von der Schweiz mit 20,4\% (von 9,3\% BIP-Anteil auf 11,2\%). Einige weitere Werte: USA $12,3 \%$, Grossbritannien 11,6\%, Deutschland 10,1\%, Frankreich 7,8\%, Österreich $2,7 \%$ und Italien 1,2\%.

\section{Gesundheitsausgaben pro Kopf}

Auch gemessen an den Gesundheitsausgaben pro Kopf der Bevölkerung haben nur die USA 
ein noch teureres Gesundheitswesen als die Schweiz: US-\$ 5267 im Jahr 2002 gegenüber US-\$ 3445 der Schweiz. Seit 1990 steht die Schweiz in jedem Jahr im zweiten Rang nach den USA mit ihren extrem hohen Gesundheitsausgaben. Für ihr Gesundheitswesen geben die USA pro Einwohner 53\% mehr aus als die Schweiz. Es folgen Norwegen, Luxemburg, Kanada, Deutschland, Island, Frankreich, die Niederlande, Dänemark und Schweden (Tab. 1, rechte Spalten). Im Jahr 2002 betrugen die durchschnittlichen Ausgaben der erfassten Länder US-\$ 2246 pro Kopf der Bevölkerung (Vorjahr: 2093).

Die Schweiz liegt 69\% (Vorjahr 57\%) höher als dieser Durchschnitt von 26 OECD-Ländern, die USA sogar 158\% (Vorjahr 133\%). Diese Dollarbeträge sind kaufkraftbereinigt, berücksichtigen also die unterschiedliche Kaufkraft in den einzelnen Ländern. Von 2001 auf 2002

Tabelle 1

Gesundheitsausgaben 2002 in US-\$ (kaufkraftbereinigt) pro Kopf sowie in \% des Bruttoinlandsprodukts BIP.

\begin{tabular}{|c|c|c|c|c|}
\hline Land* & US-\$ & Rang & $\%$ BIP & Rang \\
\hline USA & 5267 & 1 & 14,6 & 1 \\
\hline Schweiz & 3445 & 2 & 11,2 & 2 \\
\hline Norwegen & 3083 & 3 & 8,7 & 12 \\
\hline Luxemburg & 3065 & 4 & 6,2 & 19 \\
\hline Kanada & 2931 & 5 & 9,6 & 6 \\
\hline Deutschland & 2817 & 6 & 10,9 & 3 \\
\hline Island & 2807 & 7 & 9,9 & 4 \\
\hline Frankreich & 2736 & 8 & 9,7 & 5 \\
\hline Niederlande & 2643 & 9 & 9,1 & 10 \\
\hline Dänemark & 2580 & 10 & 8,8 & 11 \\
\hline Schweden & 2517 & 11 & 9,2 & 9 \\
\hline Belgien & 2515 & 12 & 9,1 & 10 \\
\hline Irland & 2367 & 13 & 7,3 & 18 \\
\hline Österreich & 2220 & 14 & 7,7 & 15 \\
\hline Italien & 2166 & 15 & 8,5 & 13 \\
\hline Grossbritannien & 2160 & 16 & 7,7 & 15 \\
\hline Finnland & 1943 & 17 & 7,3 & 18 \\
\hline Neuseeland & 1857 & 18 & 8,5 & 13 \\
\hline Griechenland & 1814 & 19 & 9,5 & 7 \\
\hline Portugal & 1702 & 20 & 9,3 & 8 \\
\hline Spanien & 1646 & 21 & 7,6 & 16 \\
\hline Tschechien & 1118 & 22 & 7,4 & 17 \\
\hline Ungarn & 1079 & 23 & 7,8 & 14 \\
\hline Slowakei & 698 & 24 & 5,7 & 21 \\
\hline Polen & 654 & 25 & 6,1 & 20 \\
\hline Mexiko & 553 & 26 & 6,1 & 20 \\
\hline Durchschnitt & 2246 & & 8,6 & \\
\hline
\end{tabular}

stiegen die Pro-Kopf-Ausgaben in der Schweiz um $6,1 \%$, in den USA um 8,1\% und im OECDDurchschnitt um 7,3\%. Die jährlichen Wachstumsraten pro Kopf im Vergleich 1997-2002: Schweiz 4,1\%, USA 6\%, OECD-Durchschnitt $5,9 \%$.

\section{Direktzahlung von Leistungen}

Eklatant ist, wieviel die Schweizer Bevölkerung direkt aus eigener Tasche bezahlen muss. Diese «out of pocket»-Zahlungen für rezeptfreie Medi-

\section{Tabelle 2}

Ärztedichte 2002. Praktizierende Ärzte je 1000 Einwohner.

\begin{tabular}{|c|c|}
\hline Griechenland $^{a}$ & 4,5 \\
\hline Italien & 4,4 \\
\hline Belgien $^{c}$ & 3,9 \\
\hline Island ${ }^{c}$ & 3,6 \\
\hline Schweiz & 3,6 \\
\hline Slowakei & 3,6 \\
\hline Tschechien $^{d}$ & 3,5 \\
\hline Norwegen $^{d}$ & 3,4 \\
\hline Dänemarkc & 3,3 \\
\hline Deutschland & 3,3 \\
\hline Frankreich ${ }^{c}$ & 3,3 \\
\hline Österreich & 3,3 \\
\hline Portugal $^{a}$ & 3,2 \\
\hline Ungarn & 3,2 \\
\hline Finnland & 3,1 \\
\hline Niederlande $^{e}$ & 3,1 \\
\hline Schweden $^{\text {b }}$ & 3,0 \\
\hline Spanien & 2,9 \\
\hline Luxemburg $^{c}$ & 2,6 \\
\hline Australien $^{a}$ & 2,5 \\
\hline Irland ${ }^{e}$ & 2,4 \\
\hline USA $^{a}$ & 2,4 \\
\hline Polen & 2,3 \\
\hline Grossbritannien & 2,1 \\
\hline Kanada & 2,1 \\
\hline Neuseeland & 2,1 \\
\hline Japan & 2,0 \\
\hline Südkorea & 1,5 \\
\hline Mexiko & 1,5 \\
\hline Türkei & 1,3 \\
\hline Durchschnitt & 2,9 \\
\hline \multicolumn{2}{|c|}{$\begin{array}{l}\text { a } 2001 ; \\
\text { b 2000; } \\
\text { c inkl. Ärzte in Industrie, Verwaltung und Forschung; } \\
\text { d Vollzeitäquivalente; } \\
\text { e alle Ärzte mit Praxisbewilligung. }\end{array}$} \\
\hline
\end{tabular}


kamente, Zahnmedizin, Pflegeheime, Spitex u. a. betragen pro Kopf US-\$ 1085 (kaufkraftbereinigt). Das sind 31,5\% der Gesamtausgaben. So hoch ist diese Selbstzahlerbelastung in keinem anderen OECD-Land, nicht einmal in den USA (37US-\$). Der Schweizer Wert ist seit Jahren rund dreimal höher als der OECD-Durchschnitt (2002: US\$ 375). Gemäss vorläufigen Zahlen des Bundesamts für Statistik für 2002 zahlten die Schweizer Fr. 12,563 Milliarden «out of pocket» $(26,2 \%$ aller Gesundheitsausgaben). Dazu kamen noch Fr. 2,54 Milliarden für Kostenbeteiligungen.

Diese extrem hohen Barauslagen der Haushalte [2] haben stark dazu beigetragen, dass die Schweiz im WHO-Vergleich der Gesundheitssysteme von 191 Ländern [3] schlecht abschnitt, jedenfalls in Relation zu ihren hohen Ausgaben. Beim Kriterium Fairness der Finanzierung erreichte sie nur den 38. bis 40. Rang und bei der Gesamtbeurteilung aller Kriterien («overall health system perfomance») nur den 20. Rang der 191 Länder [4]. Diese «Rangliste» der WHO mit den Zahlen für 1997 ist allerdings nicht unbestritten: Wer hätte auf die Ränge 1-5 getippt (Frankreich, Italien, San Marino, Andorra und Malta)? Man muss deshalb bei der Lektüre dieser WHO-Studie genau beachten, wie die einzelnen Kriterien definiert, gemessen und gewichtet wurden.

\section{Hohe Ärztedichte}

Die CD-ROM enthält für 2002 eine Statistik der Ärztedichte aller 30 OECD-Länder (Tab. 2). Danach ist die Schweiz bezüglich praktizierender Ärzte je 1000 Einwohner zusammen mit Island und der Slowakei im 4. Rang nach Griechenland, Italien und Belgien. Unsere Ärztedichte ist 24\% höher als der Durchschnitt dieser 30 Länder. Zu beachten sind allerdings die Anmerkungen zur Tabelle, da die Vergleichbarkeit internationaler Statistiken immer noch unterentwickelt ist. (Im Zwergstaat Schweiz ist es allerdings noch schlimmer, da sind die Werte oft nicht einmal zwischen den Kantonen oder Gemeinden vergleichbar ...)

Die CD-ROM enthält zahlreiche weitere Statistiken zu Gesundheitszustand, Ressourcen im Gesundheitswesen, Inanspruchnahme, Finanzierung, Versicherungen, Pharmamarkt, nichtmedizinischen Gesundheitsdeterminanten sowie demographischen und ökonomischen Grunddaten.

Vor einigen Monaten hat die OECD eine weitere, ausgezeichnete Studie publiziert: «Towards High-Performing Health Systems» [5]. Es ist eine Zusammenfassung verschiedener Untersuchungen der OECD in den letzten Jahren zu den Themen Gesundheitszustand, Zugang zur Gesundheitsversorgung, Zufriedenheit mit dem Gesundheitswesen, Kosten, Finanzierung und Effizienz. Leider ist festzustellen, dass die ausländischen Erfahrungen und Studien für die nationale Gesundheitspolitik zuwenig ausgewertet werden. Dies ist einer der Gründe für die eklatante Reformschwäche im Gesundheitswesen.

\section{Literatur}

1 Die CD-ROM «OECD Health Data 2004» kostet $325 €$ (nichtkommerzielle Organisationen 225€) und ist sechssprachig englisch/französisch/ deutsch/italienisch/spanisch/russisch. Informationsmaterial und eine Demoversion mit älteren Zahlen können kostenlos abgerufen werden unter www.oecd.org.

2 Schneider M et al. Zuzahlungen im internationalen Vergleich. Augsburg: Basys; 2004.

3 WHO. Health Systems: Improving Performance. The World Health Report 2000. Genf: WHO; 2000. Kostenlos abrufbar unter www.who.int/ whr/previous/en/. Auch in französisch erschienen.

4 Oggier W. Internationale Vergleiche. In: Kocher G, Oggier W (Hrsg.). Gesundheitswesen Schweiz 2004-2006 - Eine aktuelle Übersicht. Bern: Hans Huber; 2004. Die französische Ausgabe erscheint im Herbst.

5 OECD. Towards High-Performing Health Systems. Paris: OECD; 2004. Französische Ausgabe: Vers des systèmes de santé plus performants. 